\title{
Validación de un cuestionario para medir el estilo de vida de los estudiantes Universitarios en el marco de la teoría de Nola Pender (EVEU)
}

MSc. Marta Lorena Espinoza Lara Centro de Investigación y Estudios de la Salud (CIES)

UNAN-Managua mespinoza@unan.edu.ni

Tutor: Dr. Jairo Vanegas López Facultad de Ciencias Médicas, Santiago de Chile Universidad de Santiago de Chile https://doi.org/10.5377/torreon.v7i19.7909

Palabras clave: estilo de vida, autoestima, estudiantes universitarios, confiabilidad, validez, instrumento.

\section{RESUMEN}

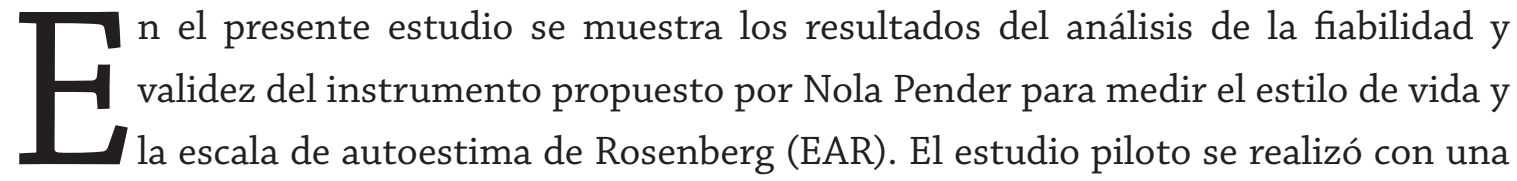
muestra de 60 estudiantes del Recinto Universitario Rubén Darío. Los resultados confirman una alta consistencia interna y una satisfactoria fiabilidad temporal. Asimismo los datos apoyan la validez (de constructo y de grupos conocidos) del instrumento. La facilidad de aplicación de esta prueba y sus aceptables características psicométricas constituyen un importante apoyo para su utilización en contextos universitarios.

\section{INTRODUCCIÓN}

Por Estilos de vida se entiende la "manera general de vivir" de las personas y grupos. Esto incluye no sólo los clásicamente denominados "hábitos saludables" (comer sano, hacer ejercicio 
físico, uso moderado del alcohol, vida sexual sana, conducción segura, manejo del estrés, no fumar) sino también las formas de pensar y de comportarse de las personas en su relación consigo mismas, en el control de sus vidas y su proyecto de vida, las relaciones interpersonales, la relación con el entorno, el repertorio de habilidades personales y sociales de que disponen, etc. (Ozcoidi \& Pérez Jarauta, Febrero 2002)

Es importante reconocer que no existe un estilo de vida "óptimo" al que puedan adscribir todas las personas. La cultura, los ingresos, la estructura familiar, la edad, la capacidad física, el entorno doméstico y laboral, harán más atractivas, factibles y adecuadas determinadas formas y condiciones de vida. Por ello, la estrategia de crear ambientes favorables para la salud se centra en gran medida en la necesidad de mejorar y cambiar las condiciones de vida para apoyar la salud (OMS, 1998).

Existen algunos estudios sobre estilos de vida en el que se integran los dominios de conductas y preferencias relacionadas con el tipo de alimentación, actividad física, consumo de alcohol, tabaco u otras drogas, responsabilidad para la salud, actividades recreativas, relaciones interpersonales, prácticas sexuales, actividades laborales y patrones de consumo. (LópezCarmona, Ariza-Andraca, Rodríguez-Moctezuma, \& Munguía Miranda, 2003)

Los pocos instrumentos disponibles para medir el estilo de vida son de tipo genérico, es decir, construidos para aplicarse a población general y no a estudiantes universitarios. Dos de los cuestionarios más conocidos de este tipo son el FANTASTIC10 y el Health-Promoting Lifestyle Profile (HPLP).

El instrumento HPLP fue validado al español por Walker y colaboradores sobre una muestra de 541 adultos hispanos residentes en Estados Unidos, fundamentalmente de origen mejicano y con edades comprendidas entre 18 y 81 años, mostrando adecuadas propiedades para la valoración de las conductas promotoras de salud que integran un estilo de vida saludable, con un índice de consistencia interna de alfa de Cronbach para la escala completa de 0.94, y con un rango de 0.69 para la dimensión "manejo del estrés" a 0.82 para las de "actividad física" y "crecimiento espiritual". Las subescalas de manejo del estrés, relaciones interpersonales y responsabilidad de la salud tuvieron un alfa de $0.68,0.77$ y 0.78 respectivamente. (Casal, 2014)

La escala de autoestima de Rosenberg se trata de una de las escalas más utilizadas para la medición global de la autoestima. Desarrollada originalmente por Rosenberg (1965) para la evaluación de la autoestima en adolescentes, incluye diez ítems cuyos contenidos se centran en los sentimientos de respeto y aceptación de sí mismo/a. La mitad de los ítems están enunciados positivamente y la otra mitad negativamente. Aunque inicialmente fue diseñada como escala de Guttman, posteriormente se ha hecho común su puntuación como escala tipo Likert, donde los ítems se responden en una escala de cuatro puntos ( $1=$ muy de acuerdo, $2=$ de acuerdo, $3=$ =en 
desacuerdo, 4=totalmente en desacuerdo). De esta manera, la puntuación total de la escala fluctúa en un rango de 10 a 40, indicando la puntuación 10 la más baja autoestima y la puntuación 40 la más alta. (Morejòn, Jimènez Bòveda, \& Vàsquez Morejòn Jimènez , 2004).

Es importante resaltar que en nuestro contexto no existen instrumentos diseñados para medir los estilos de vida en universitarios, un instrumento construido para este fin puede facilitar la identificación y medición de los componentes del estilo de vida para proporcionar consejería individual de manera adecuada y oportuna.

El presente trabajo investigativo tiene por objetivo estudiar la fiabilidad y validez de una adaptación del cuestionario para medir el estilo de vida de Nola Pender y la escala de autoestima de Rosenberg (EAR) en una muestra de 60 estudiantes del Recinto Universitario Rubén Darío.

\section{MATERIAL Y MÉTODO}

Después de revisar la literatura sobre la metodología para la construcción y validación de cuestionarios, conceptos y definición del estilo de vida, dominios que lo comprenden, y su asociación con factores personales, se elaboró la primera versión de un instrumento de autoadministración para medir el estilo de vida en estudiantes universitarios.

Tras someter el instrumento a la consulta y juicio de expertos, el cuestionario reunió los criterios de calidad, se apegó al diseño de una prueba de validación. Los criterios evaluados fueron la relevancia, la coherencia, y la claridad con la que están redactados los ítems.

$\mathrm{Al}$ ser sometido a diferentes procedimientos, los jueces eliminaron aspectos irrelevantes y apoyaron la sugerencia de incorporar aquellos elementos que resultan imprescindibles para este instrumento y modificar aquellos ítems que lo requerían.

Esta primera versión del instrumento también se aplicó a un grupo piloto de 60 estudiantes universitarios para evaluar el nivel de comprensión, legibilidad y reproducibilidad.

Una vez concluida la revisión del instrumento por el grupo de expertos y efectuada la prueba piloto, se hicieron modificaciones en la redacción del ítem de aspectos personales, eliminando 18 ítems.

El instrumento final para su aplicación a la muestra de sujetos consta de 73 preguntas divididas en 3 sesiones para medir el estilo de vida y el sentimiento de satisfacción que la persona tiene de sí misma. La sesión I incluye datos personales del estudiante (sexo, edad, peso estatura, estado civil, procedencia, número de hijos, enfermedades diagnosticadas, becario estudiantil, discapacidad, facultad, carrera, año que curso, año de ingreso). La sesión II incluye el perfil de estilos de vida dividido en seis dimensiones (nutrición, ejercicio, responsabilidad en salud, manejo del estrés, soporte interpersonal, auto actualización). La sesión III cuenta con diez 
apartados redactados positivamente y cinco redactados negativamente con objeto de minimizar el efecto aquiescencia.

Para la evaluación de la consistencia interna se utilizó el alfa cronbach, y la validación de constructo se realizó a través de un análisis factorial.

También se incluyó la carta de consentimiento informado (ver Anexo A), con el fin de que el estudiante autorice su participación y se encuentre enterado de que sus datos serán utilizados exclusivamente con fines de investigación y validación, además de ser tratados de manera confidencial.

\section{RESULTADOS}

Sesenta estudiantes de pregrado del RURD de la UNAN Managua fueron susceptibles de ser participantes de la validación. El instrumento contiene una sección que reúne información personal, con el fin de obtener las características personales de la población estudiantil participante.

La información personal de los estudiantes incluyó $36.67 \%$ mujeres de procedencia urbana y un $43.33 \%$ hombres de procedencia urbana. (Ver figura 1).

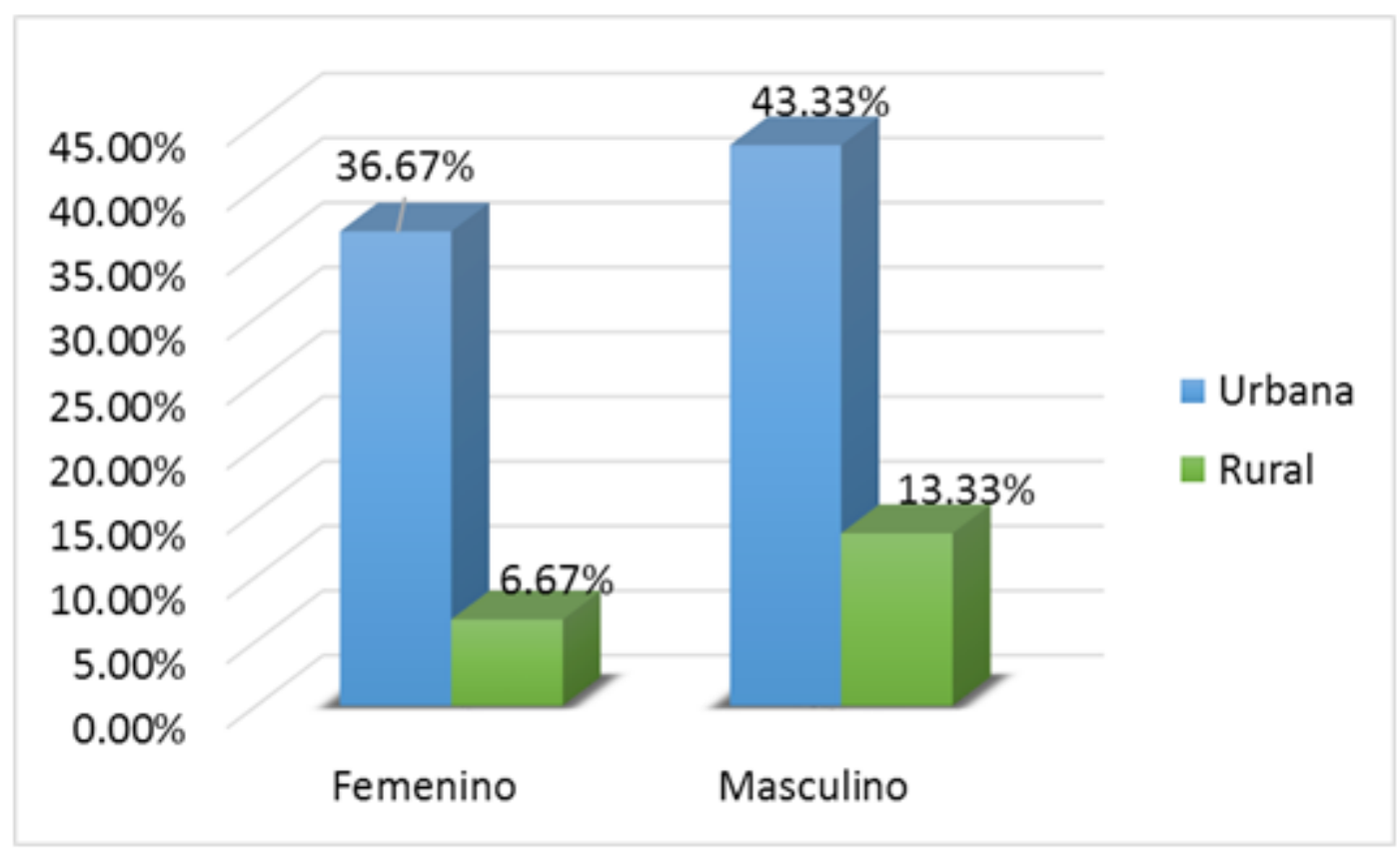

Figura 1. Sexo según procedencia de los estudiantes del RURD UNAN-Managua. Fuente: encuesta a estudiantes.

Así mismo en lo que respecta a la distribución de estudiantes por grupo de edades la muestra estuvo representada por el 62\% de estudiantes del sexo masculino en edades de 17- 20 años, 32\% en edades de 21-25 años, y el 6\% en el rango de 26-27 años. Algo semejante ocurre con sexo femenino los rangos de edades de 17-20 años es del 61\%, en el rango de 21-25 años corresponde el 35\% y en el rango de 26-27 años el $4 \%$. 
Tabla 1. Índice de masa corporal según sexo y procedencia

\begin{tabular}{|c|c|c|c|c|c|c|c|c|}
\hline & \multicolumn{4}{|c|}{ Femenino } & \multicolumn{4}{|c|}{ Masculino } \\
\hline & Urbana & Porcentaje & Rural & Porcentaje & Urbana & Porcentaje & Rural & Porcentaje \\
\hline Bajo peso & 1 & 4.54 & 0 & & 4 & 15 & 1 & 12.5 \\
\hline $\begin{array}{l}\text { Peso } \\
\text { normal }\end{array}$ & 17 & 77.27 & 3 & 75 & 12 & 46.15 & 5 & 64 \\
\hline Sobrepeso & 3 & 13.6 & 1 & 25 & 7 & 27 & 1 & 12 \\
\hline $\begin{array}{l}\text { Obesidad } \\
\text { grado } 1\end{array}$ & 0 & & 0 & & 0 & & 0 & \\
\hline $\begin{array}{l}\text { Obesidad } \\
\text { grado } 2\end{array}$ & 0 & & 0 & & 0 & & 0 & \\
\hline $\begin{array}{l}\text { Obesidad } \\
\text { grado } 3\end{array}$ & 1 & 4.54 & 0 & & 3 & 12 & 1 & 12 \\
\hline Total & 22 & 100 & 4 & 100 & 26 & 100 & 8 & 100 \\
\hline
\end{tabular}

Fuente: Encuesta estudiantes

En relación al índice de Masa Corporal (IMC) según sexo y procedencia la población femenina del área urbana se encontraba en un $77.27 \%$ en peso normal y un $13.6 \%$ en sobrepeso, de igual manera la población del área rural en un $75 \%$ se encontraba en peso normal. El sexo masculino de procedencia urbana el $46 \%$ se encontraba en peso normal, el $27 \%$ en sobrepeso, el $12 \%$ en obesidad grado 3 , el $15 \%$ en bajo peso. En cuanto a la población rural el $64 \%$ con peso normal, $12 \%$ con sobrepeso, $12 \%$ con obesidad grado $1,12 \%$ obesidad grado 3 . Del total de estudiantes seleccionados el $57 \%$ no posee ningún tipo de beca estudiantil, el $15 \%$ es becado interno, y un $17 \%$ es becado externo.

Así mismo el estilo de vida de la población participante se muestra en la tabla 2, en la que se observa que un $53.3 \%$ de los estudiantes se encuentran con un estilo de vida inadecuado y un $46 \%$ con estilo de vida adecuado.

Tabla 2. Estilo de vida estudiantes RURD

\begin{tabular}{|l|c|c|}
\hline & Frecuencia & Porcentaje \\
\hline Inadecuado estilo de vida & 32 & 53.3 \\
\hline Adecuado estilo de vida & 28 & 46.7 \\
\hline Total & 60 & 100.0 \\
\hline
\end{tabular}

Fuente: Encuesta estudiantes

En relación al estilo de vida por sexo y carrera de los estudiantes encuestados se muestra en la tabla 3 , en el que 16 estudiantes del sexo femenino que representa un $27 \%$ de los encuestados 
tienen un estilo de vida inadecuado, de igual manera 16 estudiantes del sexo masculino que representan un $27 \%$ tienen estilo de vida inadecuado. La carrera que presenta mayor índice de estilo de vida inadecuado en el sexo femenino es medicina correspondiendo a 3 estudiantes y representa a un $5 \%$, y en lo que respecta al sexo masculino 5 estudiantes equivalentes a $8 \%$ de la carrera de topografía son los que presentan estilo de vida inadecuado. (Ver tabla 3).

Tabla 3. Estilo de vida por sexo y carrera estudiantes RURD

\begin{tabular}{|c|c|c|c|c|c|c|c|c|c|c|}
\hline & \multirow{2}{*}{\multicolumn{8}{|c|}{ Sexo }} & \\
\hline & & & & & & & & & & \\
\hline & \multicolumn{4}{|c|}{ Femenino } & \multicolumn{4}{|c|}{ Masculino } & & \\
\hline & \multicolumn{2}{|c|}{$\begin{array}{l}\text { Inadecuado } \\
\text { estilo de vida }\end{array}$} & \multicolumn{2}{|c|}{$\begin{array}{c}\text { Adecuado } \\
\text { estilo de vida }\end{array}$} & \multicolumn{2}{|c|}{$\begin{array}{l}\text { Inadecuado } \\
\text { estilo de vida }\end{array}$} & \multicolumn{2}{|c|}{$\begin{array}{c}\text { Adecuado } \\
\text { estilo de vida }\end{array}$} & \multicolumn{2}{|c|}{ Total } \\
\hline Administración de empresas & 1 & $2 \%$ & 0 & $0 \%$ & 0 & $0 \%$ & 0 & $0 \%$ & 1 & $2 \%$ \\
\hline Anestesia y Reanimación & 2 & $3 \%$ & 0 & $0 \%$ & 2 & $3 \%$ & 1 & $2 \%$ & 5 & $8 \%$ \\
\hline Banca y finanzas & 0 & $0 \%$ & 0 & $0 \%$ & 1 & $2 \%$ & 2 & $3 \%$ & 3 & $5 \%$ \\
\hline Bioanalisis Clínico & 1 & $2 \%$ & 0 & $0 \%$ & 0 & $0 \%$ & 2 & $3 \%$ & 3 & $5 \%$ \\
\hline Contaduría pública y finanzas & 2 & $3 \%$ & 0 & $0 \%$ & 0 & $0 \%$ & 0 & $0 \%$ & 2 & $3 \%$ \\
\hline Enfermería CC & 1 & $2 \%$ & 1 & $2 \%$ & 2 & $3 \%$ & 2 & $3 \%$ & 6 & $10 \%$ \\
\hline Física matemática & 0 & $0 \%$ & 1 & $2 \%$ & 3 & $5 \%$ & 0 & $0 \%$ & 4 & $7 \%$ \\
\hline Fisioterapia & 2 & $3 \%$ & 1 & $2 \%$ & 0 & $0 \%$ & 2 & $3 \%$ & 5 & $8 \%$ \\
\hline Gerencia ambiental & 2 & $3 \%$ & 1 & $2 \%$ & 1 & $2 \%$ & 1 & $2 \%$ & 5 & $8 \%$ \\
\hline Matemática & 0 & $0 \%$ & 0 & $0 \%$ & 1 & $2 \%$ & 1 & $2 \%$ & 2 & $3 \%$ \\
\hline Medicina & 3 & $5 \%$ & 2 & $3 \%$ & 0 & $0 \%$ & 4 & $7 \%$ & 9 & $15 \%$ \\
\hline Mercadotecnia & 0 & $0 \%$ & 0 & $0 \%$ & 1 & $2 \%$ & 1 & $2 \%$ & 2 & $3 \%$ \\
\hline Microbiología & 1 & $2 \%$ & 0 & $0 \%$ & 0 & $0 \%$ & 0 & $0 \%$ & 1 & $2 \%$ \\
\hline Nutrición & 1 & $2 \%$ & 4 & $7 \%$ & 0 & $0 \%$ & 0 & $0 \%$ & 5 & $8 \%$ \\
\hline Topografía & 0 & $0 \%$ & 0 & $0 \%$ & 5 & $8 \%$ & 2 & $3 \%$ & 7 & $12 \%$ \\
\hline Total & 16 & $27 \%$ & 10 & $17 \%$ & 16 & $27 \%$ & 18 & $30 \%$ & 60 & $100 \%$ \\
\hline
\end{tabular}

Fuente: Encuesta estudiantes

Como puede apreciarse la figura 2 muestra la autoestima de los estudiantes encuestados en la que solamente un $2 \%$ de los estudiantes presentan una baja autoestima y en caso específico corresponde al sexo masculino. 


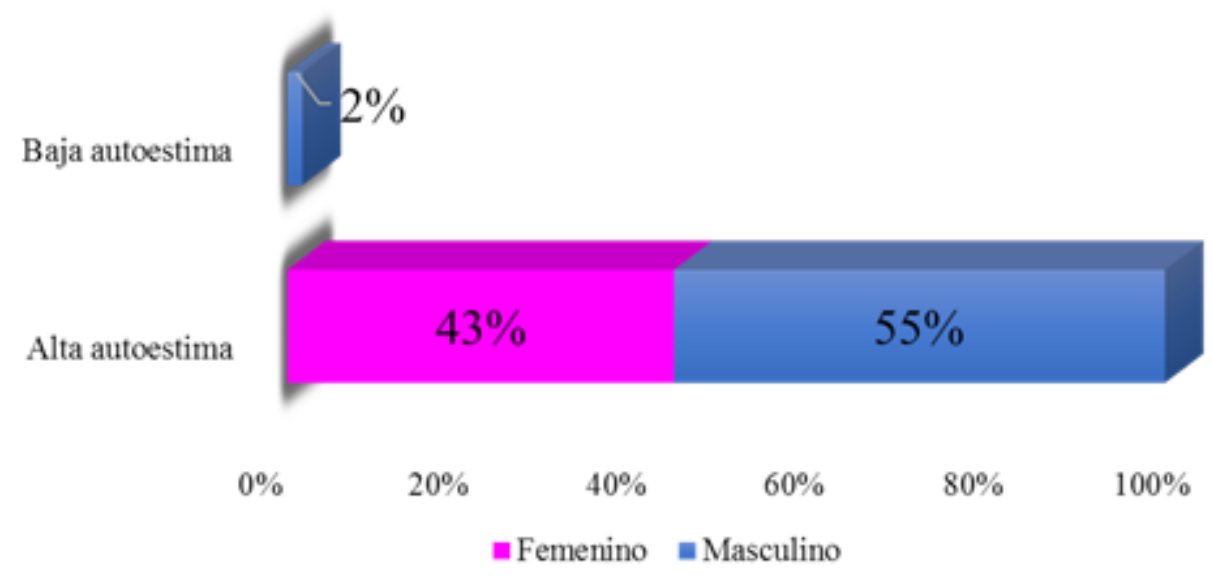

Figura 2. Autoestima de estudiantes del RURD UNAN-Managua

Se realizó el análisis de consistencia interna de las siete dimensiones incluidas en el cuestionario de Estilo de Vida Estudiantes Universitarios (EVEU); la tabla 4 muestra el coeficiente alfa de Cronbach total del instrumento fue 0.908 superando el valor de 0.70 (valor mínimo sugerido por Frías-Navarro, 2004). Considerándose excelente para ser utilizado en investigaciones que deseen medir estilo de vida, El número de elementos corresponde al número de preguntas consideradas en el instrumento. De igual manera la tabla 2 se muestra la confiabilidad por dimensiones.

Tabla 4. Estadístico de fiabilidad

\begin{tabular}{|c|c|c|}
\hline Alfa de Cronbach & $\begin{array}{c}\text { Alfa de Cronbach basada en los } \\
\text { elementos tipificados }\end{array}$ & N de elementos \\
\hline 0,908 & 0,908 & 60 \\
\hline
\end{tabular}

Fuente: Encuesta estudiantes

Tabla 5. Estadístico de fiabilidad por Dimensiones

\begin{tabular}{|l|c|c|}
\hline \multicolumn{2}{|c|}{ Dimensiones } & \multirow{2}{*}{ N de elementos } \\
\cline { 1 - 2 } Alfa de Cronbach basada en los elementos tipificados & 6 \\
\hline Nutrición & 0.776 & 5 \\
\hline Ejercicio & 0.700 & 9 \\
\hline Responsabilidad en Salud & 0.765 & 6 \\
\hline Manejo del estrés & 0.684 & 9 \\
\hline Soporte Interpersonal & 0.813 & 13 \\
\hline Auto actualización & 0.883 & 10 \\
\hline Autoestima & 0.794 & \\
\hline
\end{tabular}

Fuente: Encuesta estudiantes 
Para verificar la validez de constructo del instrumento, se estudió la medida de adecuación muestral de Kaiser-Meyer-Olkin (KMO), que es un índice que compara la magnitud de los coeficientes de correlación observados con la magnitud de los coeficientes de correlación parcial, mostrando si el instrumento cuenta o no con validez de constructo. Se realizó un análisis factorial con el método de normalización oblicuo KMO. Este método analizó la medida de adecuación muestral de Kaiser-Meyer-Olkin y proporcionó un resultado de .811 , por lo tanto es mayor que 0.5 esto nos indica que es aceptable para el análisis factorial y mientras más se acerca al 1 es mejor.

La prueba de esfericidad de Bartlett (.000) nos dice que no es significativa la hipótesis nula de variables iniciales incorrelacionadas, por lo tanto es menor que 0.05 con lo cual es adecuado el realizar el análisis factorial. Tabla 6.

Tabla 6. Prueba de KMO y Bartlett

\begin{tabular}{|c|c|c|}
\hline \multicolumn{2}{|c|}{$\begin{array}{c}\text { Medida Kaiser-Meyer-Olkin de adecuación de } \\
\text { muestreo }\end{array}$} & .811 \\
\hline \multirow{2}{*}{$\begin{array}{c}\text { Prueba de esfericidad de } \\
\text { Bartlett }\end{array}$} & Aprox. Chi-cuadrado & 184.974 \\
\cline { 2 - 3 } & Gl & 21 \\
\cline { 2 - 3 } & Sig. & .000 \\
\hline
\end{tabular}

Fuente encuesta estudiantes

Se puede observar en la tabla 7 que existen componentes que quedan bastante bien representados con la solución factorial como los son autoestima, 0.848, manejo estrés 0.760 , soporte interpersonal 0.710 . Y otros ítems que están menos representados.

Tabla 7. Comunalidades

\begin{tabular}{|l|c|c|}
\hline & Inicial & Extracción \\
\hline Nutrición & 1.000 & .657 \\
\hline Ejercicio & 1.000 & .389 \\
\hline Responsabilidad en Salud & 1.000 & .694 \\
\hline Manejo Estrés & 1.000 & .760 \\
\hline Soporte Interpersonal & 1.000 & .710 \\
\hline Auto Actualización & 1.000 & .709 \\
\hline Autoestima Rosenberg & 1.000 & .848 \\
\hline Método de extracción: análisis de componentes principales. \\
\hline
\end{tabular}

Fuente: encuesta estudiantes

La tabla 8 muestra que se identificaron dos componentes principales que explican el $68 \%$ de la varianza de las variables originales. 
Tabla 8. Varianza total explicada

\begin{tabular}{|c|c|c|c|c|c|c|c|c|c|}
\hline \multirow{2}{*}{ Componente } & \multicolumn{3}{|c|}{ Auto valores iniciales } & \multicolumn{3}{|c|}{$\begin{array}{l}\text { Sumas de extracción de } \\
\text { cargas al cuadrado }\end{array}$} & \multicolumn{3}{|c|}{$\begin{array}{l}\text { Sumas de rotación de } \\
\text { cargas al cuadrado }\end{array}$} \\
\hline & Total & $\begin{array}{c}\text { \% de } \\
\text { varianza }\end{array}$ & $\begin{array}{l}\% \text { acu- } \\
\text { mulado }\end{array}$ & Total & $\begin{array}{c}\text { \% de } \\
\text { varianza }\end{array}$ & $\begin{array}{l}\% \text { acu- } \\
\text { mulado }\end{array}$ & Total & $\begin{array}{c}\% \text { de } \\
\text { varianza }\end{array}$ & $\begin{array}{l}\% \text { acu- } \\
\text { mulado }\end{array}$ \\
\hline 1 & 3.742 & 53.456 & 53.456 & 3.742 & 53.456 & 53.456 & 3.446 & 49.222 & 49.222 \\
\hline 2 & 1.025 & 14.641 & 68.097 & 1.025 & 14.641 & 68.097 & 1.321 & 18.876 & 68.097 \\
\hline 3 & .806 & 11.511 & 79.608 & & & & & & \\
\hline 4 & .578 & 8.259 & 87.868 & & & & & 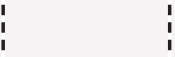 & \\
\hline 5 & .393 & 5.620 & 93.488 & & & & & & \\
\hline 6 & .240 & 3.430 & 96.918 & & & & & & \\
\hline 7 & .216 & 3.082 & 100.000 & & & & & 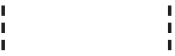 & \\
\hline
\end{tabular}

Fuente: encuesta estudiantes

\section{DISCUSIÓN}

Los resultados de validación anteriormente presentados se relacionan con los encontrados por Walker y colaboradores mostrando adecuadas propiedades para la valoración de las conductas promotoras de salud que integran un estilo de vida saludable. De igual manera el estudio realizado por (Jaimes \& Gómez Díaz, 2014), mostrando que el instrumento tiene el coeficiente de fiabilidad para la escala total de 0,93 y test - retest fiabilidad de 0,86; de manera similar los coeficientes alfa de Cronbach de las subescalas oscilaron desde 0,70 hasta 0,87.

En cuanto a la población estudiantil encuestada en la prueba piloto, presentan características personales similares a estudios relacionados a factores de riesgo de la población juvenil, encontrando predominio de estudiantes del sexo masculino proveniente del área urbana, en edad promedio de 17- 20 años, en cuanto al índice de masa corporal se encentran en sobrepeso, y obesidad grado 1,2 , y 3 , con resultados similares a los encontrados en el estudio (Jaimes \& Gómez Díaz, 2014), Por consiguiente el estilo de vida de la mayoría de los estudiantes es inadecuado, siendo las carreras de medicina y topografía las que presentan mayores porcentajes.

Con respecto a la autoestima se encontró que la mayoría de los estudiantes tienen una alta autoestima.

Los resultados demuestran la validez del cuestionario, por lo cual puede ser aplicado a muestras similares de estudiantes universitarios. 


\section{CONCLUSIONES}

Este es el primer estudio para validar el estilo de vida promotor de salud en estudiantes universitarios en nuestro contexto, sometiendo la encuesta a juicio de expertos, teniendo en cuenta que ya ha sido validada en otros países como España, Estados Unidos, en Colombia, México, en pacientes diabéticos, hipertensos. En aras de esta nueva versión del EVEU sea similar al original el proceso de validación ha seguido lo recomendado por el doctor José Supo (Supo, 2013).

El diseño de instrumentos y sus correspondientes ítems, ya sea para evaluación o pruebas deben pasar por todo el proceso anterior para asegurar que la información que se obtenga sea válida y permita una efectiva toma de decisiones.

Los ítems deben tener como punto de partida un constructo teórico, respaldado por jueces expertos y análisis estadísticos que validen los instrumentos, de lo contrario, se estaría induciendo a ofrecer soluciones incongruentes o que puedan afectar a una población estudiantil.

\section{BIBLIOGRAFÍA}

Alligood, M. R., \& Marriner Tomey, A. (2011). Modelos y Teorias en Enfermerìa. En T. J. Sakraida, Nola J. Pender (págs. 434-453). España: ELSEVIER Mosby.

Cazal, R. p. (2014). Validación de un instrumento que facilite el proceso de atención de enfermería en el marco de la Teoría de Pender. España: Universidad Da Caruña.

Frías-Navarro, D. (2014). Análisis de fiábilidád de lás puntuáciones de un instrumento de medidá. Alfá de Cronbách: un coeficiente de fiábilidád. Valencia: Universidad de Valencia. Obtenido de www. uv.es/ friasnav/AlfaCronbach.pdf

Jaimes, E. L., \& Gómez Díaz, M. P. (2014). Estilos De Vida Saludables en Estudiantes Universidad Colombia. Hacia la Promoción de la Salud, 68-83.
Morejòn, A. J., Jimènez Bòveda, R., \& Vàsquez Morejòn Jimènez , R. (2004). Escala de autoestima de Rosenberg: fiabilidad y validez en población clínica Española. Apuntes de Psicologìa, 247-255.

Osorio, A. G., Toro Rosero, M. Y., Macías Ladino, A. M., Valencia Garcés, C. A., \& Palacio Rodríguez, S. (2010). La Promociòn de la Salud Como Estrategìa para el Fomento de Estilos de Vida Saludables. Hacia la Promoción de la Salud, 128-143.

Ozcoidi, M. E., \& Pérez Jarauta, M. J. (Febrero 2002). Estilos de vida. Promoción de salud. Acciones colectivas y comunitarias. Gobierno de Navarra: Gráficas Alzate.

Pender, N. J. (2011). Heath Promotion Model Manual. University of Michigan. 
Rodríguez, A. M. (2014). Diseño y validación de instrumentos de mediciòn. Dialogos, 19-40.

Supo, J. (2013). Cómo validar un instrumento.

Perù : Copyright.
Yolanda Quiles Marco, M. Q. (2015). Promoción de la Salud: Experiencias Prácticas. Elche, España: editoreal electrònica umh.

\section{ANEXO A}

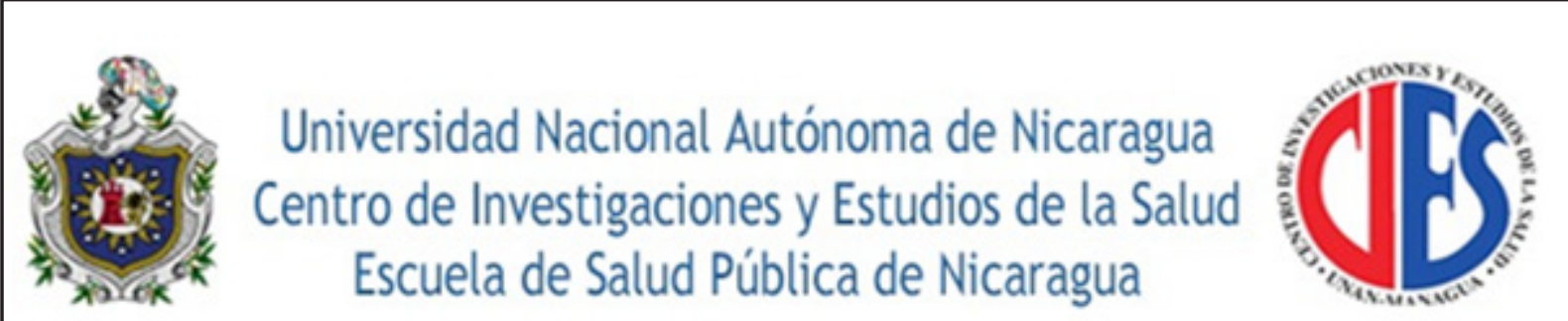

\section{Consentimiento informado}

\section{Título del proyecto}

Estilos de Vida saludables y su asociación con factores personales en Estudiantes Universitarios UNAN-Managua 2017.

\section{Investigadora}

MSc. Marta Lorena Espinoza estudiante del doctorado en Ciencias de la Salud del Centro de Investigaciones y Estudios de la Salud, CIES- UNAN- Managua.

\section{Introducción / Propósito}

El objetivo del estudio es determinar si existe asociación entre los estilos de vida y factores personales, socio familiares y sociales en los estudiantes universitarios UNANMANAGUA 2017

Con el propósito de sensibilizar a los estudiantes sobre la importancia de la adopción de estilos de vida saludables que permitan tener un estado nutricional adecuado y que repercutan positivamente en la salud de cada uno de los participantes del estudio; de la misma manera se podrá elaborar estrategias encaminadas a la adopción de estilos de vida saludables en estudiantes universitarios.

\section{Participación}

Participaran los estudiantes de todas las facultades del Recinto Universitario Rubén Darío. 


\section{Procedimientos}

Se le aplicará una encuesta sobre estilos de vida que sólo tomara un tiempo aproximado de 25 a 30 minutos.

\section{Riesgos / incomodidades}

No habrá ninguna consecuencia desfavorable para usted, en caso de no aceptar la invitación. No tendrá que hacer gasto alguno durante el estudio.

\section{Beneficios}

El beneficio que obtendrá por participar en el estudio, es el de recibir información oportuna y actualizada sobre los estilos de vida y el estado nutricional de los estudiantes universitarios.

\section{Confidencialidad de la información}

Los datos que se obtengan a lo largo del presente estudio son totalmente confidenciales, de modo que sólo se emplearán para cumplir los objetivos antes descritos. No se publicarán nombres de ningún tipo. Así que podemos garantizar confidencialidad absoluta.

\section{Consentimiento / Participación voluntaria}

Acepto participar en el estudio: He leído la información proporcionada, o me ha sido leída. He tenido la oportunidad de preguntar dudas sobre ello y se me ha respondido satisfactoriamente. Consiento voluntariamente participar en este estudio y entiendo que tengo el derecho de retirarme en cualquier momento de la entrevista sin que me afecte de ninguna manera.

\section{Nombres y firmas del participante}

Nombre y firma del participante: 